\section{RMD Open}

Rheumatic \& Musculoskeletal Diseases

\title{
Cardiovascular outcomes in systemic sclerosis with abnormal cardiovascular MRI and serum cardiac biomarkers
}

Raluca B Dumitru, ${ }^{1,2}$ Lesley-Anne Bissell,, ${ }^{1,2}$ Bara Erhayiem, ${ }^{3}$ Ananth Kidambi, ${ }^{3,4}$ Ana-Maria H Dumitru, ${ }^{1,5}$ Graham Fent, ${ }^{3}$ Giuseppina Abignano, ${ }^{1,2}$ Helena Donica, ${ }^{6}$ Agata Burska, ${ }^{1,2}$ John P Greenwood, ${ }^{3,4}$ John Biglands, ${ }^{2}$ Dominik Schlosshan, ${ }^{4}$ Francesco del Galdo, ${ }^{1,2}$ Sven Plein, ${ }^{3,4}$ Maya H Buch (ib) 1,7,8

To cite: Dumitru RB, Bissell L-A, Erhayiem B, et al. Cardiovascula outcomes in systemic sclerosis with abnormal cardiovascular MRI and serum cardiac biomarkers. RMD Open 2021;7:e001689. doi:10.1136/ rmdopen-2021-001689

Received 6 April 2021 Accepted 6 September 2021
Check for updates

(c) Author(s) (or their employer(s)) 2021. Re-use permitted under CC BY-NC. No commercial re-use. See rights and permissions. Published by BMJ.

For numbered affiliations see end of article.

Correspondence to Dr Maya H Buch; maya.buch@manchester.ac.uk

\section{ABSTRACT}

Objectives To explore the prognostic value of subclinical cardiovascular (CV) imaging measures and serum cardiac biomarkers in systemic sclerosis (SSc) for the development of CV outcomes of primary heart involvement (pHI). Methods Patients with SSc with no clinical SSc-pHI and no history of heart disease underwent cardiovascular magnetic resonance (CMR) imaging, and measurement of serum high-sensitivity-troponin I (hs-Tnl) and Nterminal-pro-brain natriuretic peptide (NT-proBNP). Follow-up clinical and CV outcome data were recorded. CV outcomes were defined as myocarditis, arrhythmia and/or echocardiographic functional impairment including systolic dysfunction and/or diastolic dysfunction.

Results Seventy-four patients with a median (IQR) age of $57(49,63)$ years, $32 \%$ diffuse cutaneous SSc, 39\% interstitial lung disease, $30 \%$ Scl70 + were followed up for median (IQR) $22(15,54)$ months. Ten patients developed CV outcomes, comprising one patient with myocarditis and systolic dysfunction and nine arrhythmias: three nonsustained ventricular tachycardia and six supraventricular arrhythmias. The probability of CV outcomes was considerably higher in those with NT-proBNP $>125 \mathrm{pg} /$ $\mathrm{mL}$ versus normal NT-proBNP $\left(X^{2}=4.47, p=0.035\right)$. Trend for poorer time-to-event was noted in those with higher extracellular volume (ECV; indicating diffuse fibrosis) and hs-Tnl levels versus those with normal values $\left(X^{2}=2.659\right.$ $p=0.103 ; X^{2}=2.530, p=0.112$, respectively). In a predictive model, NT-proBNP $>125 \mathrm{pg} / \mathrm{mL}$ associated with CV outcomes $(0 R=5.335, p=0.040)$, with a trend observed for ECV $>29 \%(0 R=4.347, p=0.073)$.

Conclusion These data indicate standard serum cardiac biomarkers (notably NT-proBNP) and CMR indices of myocardial fibrosis associate with adverse CV outcomes in SSc. This forms the basis to develop a prognostic model in larger, longitudinal studies.

\section{INTRODUCTION}

Systemic sclerosis (SSc, scleroderma) is a rare immune-mediated inflammatory disease (IMID) characterised by autoimmunity, vasculopathy and fibrosis of the skin and internal organs. It can be fatal especially

\section{Key messages}

What is already known about this subject?

- Systemic sclerosis primary heart involvement (SSc$\mathrm{pHI}$ ) is associated with increased mortality.

- Subclinical SSc-pHI, including cardiovascular magnetic resonance (CMR) and blood indicators are commonly described however, the prognostic significance is unclear.

What does this study add?

- Cardiac biomarkers, in particular N-terminal-probrain natriuretic peptide and CMR indices suggestive of diffuse myocardial fibrosis (extracellular volume), showed association with cardiovascular (CV) outcomes.

How might this impact on clinical practice or further developments?

- These data can inform a prognostic risk model of clinical SSc-pHI, aid early identification and prevent $\mathrm{CV}$ death in SSc.

when the lung, heart and/or kidneys are affected. ${ }^{12}$ Clinically overt SSc-primary heart involvement (SSc-pHI) (ie, not secondary to ischaemic heart disease (IHD), or pulmonary artery hypertension, PAH) typically manifests as myocarditis, conduction abnormalities and arrhythmia and/or heart failure. SSc-pHI is described in up to $20 \%-30 \%$ of patients with $\mathrm{SSc}^{34}$ and is associated with a mortality rate of up to $70 \% .{ }^{5}$ Historical autopsy studies and sensitive multiparametric imaging methods such as cardiovascular magnetic resonance (CMR) imaging suggest subclinical heart involvement in a majority of patients with SSc. ${ }^{7-10}$ Late gadolinium enhancement (LGE) CMR in SSc detects focal myocardial fibrosis that is distinct from scar caused by epicardial coronary artery disease. ${ }^{79}$ CMR with native $\mathrm{T} 1$ and extracellular volume (ECV) 
measurement indicates diffuse myocardial fibrosis and has been reported in up to $70 \%$ of patients with SSc. ${ }^{11-13}$ Finally, myocardial perfusion impairment due to coronary microvascular dysfunction has also been observed in patients with SSc. ${ }^{71}{ }^{15}$ Serum cardiac biomarkers troponin I (TnI) and N-terminal pro-brain natriuretic peptide (NT-proBNP) have similarly emerged as sensitive tools in the detection of heart involvement. ${ }^{16}$ Elevated cardiac biomarkers have been observed in patients with SSc and associated with cardiovascular (CV) events while more recent data also demonstrate their association with subclinical SSc-pHI. ${ }^{15} 17$

The prognostic implication of subclinical SSc-pHI however has not been established. Only a subgroup of patients with subclinical SSc-pHI develop clinically overt SSc-pHI. Identifying patients at risk of clinically meaningful SSc-pHI and the indices associated with progression to clinical presentation is an unmet need. We recently reported on one of the largest studies to describe subclinical CMR features of SSc-pHI, demonstrating reduced microvascular perfusion and presence of myocardial focal and diffuse fibrosis in 83 patients with SSc. ${ }^{18}$ This subclinical phenotype associated with highsensitivity (hs)-TnI and NT-proBNP and also SSc disease severity and complicated peripheral vasculopathy. We now report on the clinical and functional outcomes of this cohort. The principal aim was to explore the prognostic value of CMR and serum cardiac biomarkers for the development of CV outcomes of SSc-pHI.

\section{METHODS}

\section{Study design}

We report on patients recruited as part of two observational studies from 2013 to 2019 at the Department of Rheumatology, The Leeds Teaching Hospital NHS Trust ('Connective Tissue Disease and Vasculitis Cohort' and 'ELectrophysiology and CArdiac imaging in SclerodermA' (ELCASA) study). The studies were conducted according to the Declaration of Helsinki. All participants provided written informed consent.

\section{Patients}

All patients fulfilled the 2013 American College of Rheumatology/EULAR criteria for $\mathrm{SSc}^{19}$ and were classified as limited cutaneous SSc or diffuse cutaneous SSc (dcSSc), respectively, according to the LeRoy classification. ${ }^{20}$ Exclusion criteria included any prior diagnosis of SSc-pHI or other cardiomyopathies, arrhythmia/rhythm disturbances, valve disease, abnormal systolic or diastolic (>grade 1) function, a diagnosis of PAH, IHD, diabetes or more than two traditional CV risk factors defined as current smoker, hypertension, hypercholesterolaemia/ hypertriglyceridaemia and family history of premature CV disease (CVD). Patients with any other inflammatory musculoskeletal conditions were also excluded. All participants were followed up for a minimum of 12 months.

\section{Schedule of assessments}

\section{Clinical data}

Comprehensive demographic and clinical data were collected including SSc subtype, duration, serology, organ involvement, any prior diagnosis of heart disease, current and previous immunosuppression and nailfold capillaroscopy findings.

\section{Testing and assessments}

All patients had annual Doppler echocardiography as per standard practice with additional testing, including ECG, 24-hour Holter ECG and stress ECG if clinically indicated. The 'ELCASA' cohort ${ }^{15}$ also had an implantable loop recorder (ILR) (Medtronic Reveal XT) inserted and 24-hour Holter monitor yearly. These patients were followed for 3 years and had the ILR interrogated every 3 months up until April 2017, after which they continued with testing for the wider group as described above.

\section{Serum cardiac biomarker testing}

The whole cohort had baseline serum samples collected for hs-TnI and NT-proBNP testing, measured using the same technique. Hs-TnI was measured on a Siemens Advia XPT system (Advia Chemistry XPT and Advia Centaur XPT Immunoassay, respectively) and NT-proBNP on Cobas 6000 (immunochemistry module Cobas e601) and the kits supplied by Roche Diagnostics. According to the manufacturer recommendations, a concentration above $37 \mathrm{ng} / \mathrm{mL}$ for hs-TnI and above $125 \mathrm{ng} / \mathrm{mL}$ for NT-proBNP constituted abnormal.

\section{CMR imaging}

CMR was performed at baseline, on a 3 Tesla Philips Achieva MR system, as previously described..$^{15} 1821$ The same CMR protocol was used for the whole cohort and comprised left ventricle (LV) function and volume, LGE, native and post-contrast T1 mapping for ECV estimation and adenosine stress and rest myocardial perfusion with myocardial blood flow (MBF) and myocardial perfusion reserve (MPR) assessment. Cvi42 software (V.4.1.3, Circle Cardiovascular Imaging, Calgary, Canada) was used for CMR image analysis. LGE was reported according to the 16 segment American Heart Association (AHA) model ${ }^{22}$ and the five-SD method was used for LGE mass quantification. ${ }^{23}$ Native and post-contrast myocardial and blood pool T1 were measured in order to calculate $\mathrm{ECV}^{24}$ Perfusion was assessed visually by comparing the rest and stress perfusion images using the 16 segment AHA model. ${ }^{22}$ Quantitative analysis of the perfusion data were also performed to generate estimates of MBF at stress and rest. ${ }^{25}$ MPR values were calculated by dividing the stress by the rest MBF. According to the departmental reference ranges, an ECV $>29 \%$ and $\mathrm{T} 1$ native $>1240 \mathrm{~ms}$ were classified as abnormal.

\section{Study endpoint}

Any CV outcomes that occurred up to the end of study period were recorded. The primary endpoint of clinically relevant SSc-pHI was defined as any episode of 
myocarditis, rhythm disturbances including supraventricular, ventricular and atrioventricular block and/ or clinically relevant (echocardiographic) functional impairment including systolic dysfunction (defined as a left ventricular ejection fraction $<50 \%$ ) and/or a diastolic dysfunction $>$ grade 1 (defined as an $\mathrm{E} / \mathrm{A}$ ratio $>0.8$; deceleration time $<200 \mathrm{~ms}$ ).

\section{Statistical analysis}

Student t-test and Mann-Whitney U for continuous variables and Fisher's exact test for categorical variables were used to assess for significant between group differences. Kaplan-Meier survival curves and log-rank test were performed to plot cumulative time-to-event outcomes between groups. Univariate logistic regression was used to identify association between CV outcomes and CMR and cardiac serum biomarkers. The penalised maximum likelihood estimation method was employed to evaluate the predictive value of CMR measures and cardiac biomarkers for the development of CV outcomes. Principal components analysis was applied over all CMR variables to predict missing values in the model. With this pilot study, a two-tailed $p$ value of 0.05 was used to indicate strength of finding as opposed to assigning statistical significance. The statistical analysis was performed using SPSS (IBM SPSS Statistics 22) and R (V.3.5.2).

\section{RESULTS}

\section{Baseline characteristics}

Seventy-four patients with SSc had available baseline and follow-up outcome data and were included in the study. Eighteen of these had an ILR implanted. Participants had a median (IQR) age of $57(49,63)$ years, $24(32 \%)$ had dcSSc and 29 (39\%) had a history of interstitial lung disease (ILD). Twenty-seven (36\%) were anti-centromere antibody (ACA) positive and $22(30 \%)$ were anti-topoisomerase antibody (Scl70) positive (table 1). Forty-two (57\%) patients were receiving a disease modifying anti-rheumatic drug at the time of recruitment. Twenty-one (28\%) patients had $\mathrm{CV}$ risk factors, 16 had one $\mathrm{CV}$ risk factor and 5 had two CV risk factors. None of the patients had any rhythm disturbances, evidence of IHD on ECG or evidence of functional impairment on CMR.

\section{Cardiovascular outcomes}

Patients were followed up for a median (IQR) of 22 (15, 54) months. Eleven CV outcomes in 10 patients were recorded, comprising one diagnosis of myocarditis (new clinical presentation; associated with LV systolic dysfunction, with subsequent CMR confirmation) and patients with arrhythmias (table 2). The arrhythmias included three non-sustained ventricular tachycardia (NSVT) and six supraventricular arrhythmias; of which one patient had atrial tachycardia, three atrial fibrillation of which one also had atrial flutter, one patient with atrial flutter and another one with supraventricular tachycardia. For seven out of nine patients with SSc the arrhythmias were picked up by the ILR, only one patient being symptomatic (with
Table 1 Disease characteristics of patients with SSc

Patients with

SSc phenotype

SSc, $\mathrm{n}=74$

\begin{tabular}{|c|c|}
\hline Age, median (IQR) & $57(49,63)$ \\
\hline Female & $63(85)$ \\
\hline \multicolumn{2}{|l|}{ Disease subtype } \\
\hline IcSSc & $50(68)$ \\
\hline dcSSc & $24(32)$ \\
\hline Disease duration (years), median (IQR) & $8(2,16)$ \\
\hline \multicolumn{2}{|l|}{ History of } \\
\hline Digital ulceration & $16(22)$ \\
\hline Calcinosis & $17(23)$ \\
\hline Myositis & $3(4)$ \\
\hline GORD & $66(90)$ \\
\hline Interstitial lung disease & $29(39)$ \\
\hline Current use of DMARD & $42(57)$ \\
\hline \multicolumn{2}{|l|}{ Clinical profile } \\
\hline $\begin{array}{l}\text { Total modified Rodnan skin score, median } \\
\text { (IQR) }\end{array}$ & $2(1,6)$ \\
\hline Digital ulceration & $10(14)$ \\
\hline Tendon friction rubs & $2(3)$ \\
\hline Calcinosis & $14(19)$ \\
\hline \multicolumn{2}{|l|}{ Antibody profile } \\
\hline ANA & $69(93)$ \\
\hline ACA & $27(36)$ \\
\hline Scl70 & $22(30)$ \\
\hline \multicolumn{2}{|l|}{$\mathrm{CV}$ risk factors } \\
\hline Any CV risk factors, & $21(28)$ \\
\hline FHx premature CVD & $8(11)$ \\
\hline Smoking & $6(8)$ \\
\hline Dyslipidaemia & $3(4)$ \\
\hline HBP & $8(11)$ \\
\hline One $\mathrm{CV}$ risk factor & $16(21)$ \\
\hline Two CV risk factors & $5(7)$ \\
\hline
\end{tabular}

$\mathrm{N} \%$ presented unless stated otherwise.

ACA, anti-centromere antibody; ANA, antinuclear antibodies; $\mathrm{CV}$, cardiovascular; CVD, cardiovascular disease; dcSSc, diffuse cutaneous systemic sclerosis; DMARD, disease modifying anti-rheumatic drugs; ESR, erythrocyte sedimentation rate; FHx, family history; GORD, gastro-oesophageal reflux disease; HBP, high blood pressure; IcSSc, limited cutaneous systemic sclerosis; Scl70, anti-topoisomerase antibody; SSc, systemic sclerosis.

palpitations) at the time of the arrhythmia. Two additional patients with SSc developed atrial tachycardia and NSVT detected on 24-hour Holter ECG and stress ECG, investigations that were carried out following an episode of syncope and dizziness with palpitations, respectively.

None of the 74 patients had diastolic dysfunction >grade 1 . The median (IQR) time to CV outcome was $14(8,27)$ months. The patients with SSc with CV outcomes had a median (IQR) baseline age and disease 


\begin{tabular}{|c|c|c|c|c|c|c|c|c|c|c|}
\hline & Patient 1 & Patient 2 & Patient 3 & Patient 4 & Patient 5 & Patient 6 & Patient 7 & Patient 8 & Patient 9 & Patient 10 \\
\hline CV outcome & SVT & SVT & $\mathrm{AF}$ & AF & $\begin{array}{l}\text { AF and } \\
\text { flutter }\end{array}$ & $\begin{array}{l}\text { Atrial } \\
\text { flutter }\end{array}$ & NSVT & NSVT & NSVT & $\begin{array}{l}\text { Myocarditis } \\
\text { systolic } \\
\text { dysfunction }\end{array}$ \\
\hline Baseline ECG & - & SR & SR & SR & SR & SR & SR & SR, VE & $\begin{array}{l}\text { SR, } \\
\text { unspecific } \\
\text { flattened T } \\
\text { wave }\end{array}$ & SR \\
\hline Age (years) & 74 & 62 & 57 & 73 & 49 & 55 & 52 & 59 & 50 & 60 \\
\hline Gender & $\mathrm{F}$ & $\mathrm{F}$ & $\mathrm{F}$ & $\mathrm{F}$ & $\mathrm{F}$ & $\mathrm{F}$ & $\mathrm{F}$ & $\mathrm{F}$ & $M$ & $\mathrm{~F}$ \\
\hline SSc subset & IcSSc & IcSSc & dcSSc & IcSSc & IcSSc & IcSSc & dcSSc & dcSSc & dcSSc & dcSSc \\
\hline ILD & No & No & No & No & No & No & Yes & Yes & Yes & Yes \\
\hline MRSS & 6 & 4 & 14 & 2 & 2 & 6 & 11 & 18 & 0 & 9 \\
\hline ANA & ACA & $A C A$ & - & ACA & $A C A$ & ACA & Scl70 & Anti Ro & Scl70 & Scl70 \\
\hline History of DU & Yes & No & No & No & No & Yes & No & No & No & No \\
\hline $\begin{array}{l}\text { NT-proBNP } \\
\text { (pg/mL) }\end{array}$ & 161 & 288 & 105 & 127 & 32 & 141 & 329 & 105 & 38 & 470 \\
\hline Hs-Tnl (ng/L) & 3 & 5 & 3 & - & 6 & 31 & 313 & 5 & 3 & 225 \\
\hline
\end{tabular}

ACA, anti-centromere antibody; AF, atrial fibrillation; ANA, antinuclear antibodies; CV, cardiovascular; dcSSc, diffuse cutaneous systemic sclerosis; DU, digital ulcers; hs-Tnl, high-sensitivity troponin I; IcSSc, limited cutaneous systemic sclerosis; ILD, interstitial lung disease; MRSS, modified Rodnan skin score; NSVT, non-sustained ventricular tachycardia; NT-proBNP, N-terminal pro-brain natriuretic peptide; Scl70, anti-topoisomerase antibody; SR, sinus rhythm; SSc, systemic sclerosis; SVT, supraventricular tachycardia; VE, ventricular ectopics.

duration of $58(52,64)$ and $2(1,22)$ years, respectively, nine were female, five had dcSSc, four had a diagnosis of ILD, three were Scl70 and five were ACA positive. All patients were in sinus rhythm, and none had presentations of IHD.

\section{CV outcomes and CMR indices}

Of the 72 patients with available LGE data, 14 had focal LGE fibrosis in a non-coronary distribution. Only one of these sustained a CV outcome. The other eight patients with CV outcomes showed no LGE (available LGE data in 9 out of 10 that sustained a CV outcomes) (table 3). Seventy-one of 74 patients had available ECV data of which 9 had a CV outcome. An ECV $>29 \%$ was recorded in 8/9 patients with SSc with CV outcomes versus 40/62 patients with no CV outcomes (table 3 ). In other words, 48/71 patients with SSc had an ECV above the normal threshold $(>29 \%)$ of which 8 sustained a CV outcome compared with only $1 / 23$ patients with SSc with normal ECV (of available ECV data in 9/10 that sustained a CV outcome). Numerically higher LV mass was noted in those with CV outcomes (mean (SD) 48 (10) vs 43 (10), $\mathrm{p}=0.102$ ) (table 3 ) although within normal range. ${ }^{26}$ There was no difference in MPR between those with and without CV outcomes (median (IQR) $1.86(1.5,2.4)$ vs 1.96 (1.6, 2.5), respectively, $\mathrm{p}=0.696)$. The mean LV volume and function were within normal range, with no suggestion of impairment in those with a CV outcome.

\section{CV outcomes and serum cardiac biomarkers}

Patients with CV outcomes had modestly higher hs-TnI (median (IQR) $5(3,127)$ vs $3(3,7)$, respectively, $\mathrm{p}=0.116$ ) and higher NT-proBNP (median (IQR) $134(88,298)$ vs $83(48,124)$, respectively, $\mathrm{p}=0.114)$ compared with those with no CV outcomes. A NT-proBNP above the normal reference range $(>125 \mathrm{pg} / \mathrm{mL})$ was recorded in $6 / 10$ patients with CV outcomes versus $15 / 64$ patients with no CV outcomes $(\mathrm{p}=0.026)$ (table 3$)$. That is, CV outcomes were recorded in $6 / 10$ with NT-proBNP above the normal range $(>125 \mathrm{pg} / \mathrm{mL})$ and in $4 / 53$ patients with normal NT-proBNP levels. An abnormal hs-TnI was noted in 2/9 patients with CV outcomes versus 4/61 patients with no CV outcomes (table 3). Therefore, of the 70 patients with available hs-TnI data, CV outcomes occurred in 2/6 patients with abnormal hs-TnI $(>37 \mathrm{ng} / \mathrm{L})$ and in $7 / 64$ with normal hs-TnI levels.

\section{Probability of CV outcomes in those with and without abnormal CMR and cardiac serum biomarkers}

The probability of CV outcomes was higher in those with NT-proBNP above the normal threshold compared with those with normal NT-proBNP levels $\left(\mathrm{X}^{2}=4.47, \mathrm{p}=0.035\right)$ (figure 1). A trend in those with higher ECV compared with those with normal $\mathrm{ECV}$ values $\left(\mathrm{X}^{2}=2.659, \mathrm{p}=0.103\right)$ (figure 1) and in those with hs-TnI above the normal threshold ( $\geq 37)$ compared with those with normal hs-TnI levels was also observed $\left(\mathrm{X}^{2}=2.530, \mathrm{p}=0.112\right)$. The probability of CV outcomes did not differ between patients with LGE compared with those with no LGE $\left(\mathrm{X}^{2}=0.250\right.$, $\mathrm{p}=0.617$ ) (figure 1).

\section{CMR and cardiac biomarkers as predictors of CV outcomes}

Univariate logistic analysis showed an association of NT-proBNP above the normal threshold $(>125 \mathrm{pg} / \mathrm{mL})$ with the presence of $\mathrm{CV}$ outcomes $(\mathrm{OR}=4.90, \mathrm{p}=0.025)$. Hs-TnI also associated with the presence of CV outcomes $(\mathrm{OR}=1.009, \mathrm{p}=0.040)$. There was no association between 
Table 3 CMR parameters and cardiac biomarkers in patients with SSc with CV outcomes versus patients with SSc with no CV outcomes

\begin{tabular}{|c|c|c|c|}
\hline & $\begin{array}{l}\text { Patients with SSc with CV } \\
\text { outcomes }\end{array}$ & $\begin{array}{l}\text { Patients with SSc with no } \\
\text { CV outcomes }\end{array}$ & $P$ value \\
\hline \multicolumn{4}{|l|}{ CMR variable } \\
\hline \multicolumn{4}{|c|}{ Myocardial inflammation and fibrosis } \\
\hline ECV\% & $31(2)$ & $30(3)$ & 0.61 \\
\hline$E C V>29, n \%$ & 08 September & $40 / 62$ & Fisher's exact test 0.254 \\
\hline T1 native (ms) & $1216(88)$ & $1237(72)$ & 0.434 \\
\hline $\mathrm{T} 1$ native $>1240 \mathrm{~ms}, \mathrm{n} \%$ & $5 / 9$ & $33 / 64$ & Fisher's exact test 1 \\
\hline LGE presence & $1 / 9$ & $13 / 63$ & Fisher's exact test 0.679 \\
\hline Fibrosis mass & $0.42(1.27)$ & $0.45(1.2)$ & 0.931 \\
\hline LGE\% (from LV mass) & $0.60(1.8)$ & $0.59(1.5)$ & 0.822 \\
\hline \multicolumn{4}{|l|}{ Myocardial perfusion } \\
\hline MPR, median (IQR) & $1.86(1.5,2.4)$ & $1.96(1.6,2.5)$ & 0.696 \\
\hline Stress MBF, median (IQR) & $1.98(1.8,2.6)$ & $1.93(1.4,2.7)$ & 0.635 \\
\hline Rest MBF, median (IQR) & $1.21(0.8,1.4)$ & $0.99(0.7,1.2)$ & 0.339 \\
\hline \multicolumn{4}{|l|}{ LV dimension and function } \\
\hline LVEDV/BSA $\left(\mathrm{ml} / \mathrm{m}^{2}\right)$ & $76(15)$ & $80(14)$ & 0.408 \\
\hline LVESV/BSA $\left(\mathrm{ml} / \mathrm{m}^{2}\right)$ & $30(8)$ & $30(9)$ & 0.926 \\
\hline LVSV/BSA $\left(\mathrm{ml} / \mathrm{m}^{2}\right)$ & $50(7)$ & $47(7)$ & 0.191 \\
\hline LV mass/BSA $\left(\mathrm{g} / \mathrm{m}^{2}\right)$ & $48(10)$ & $43(10)$ & 0.102 \\
\hline LVEF \% & $63(5)$ & $62(5)$ & 0.496 \\
\hline \multicolumn{4}{|l|}{ Cardiac biomarkers } \\
\hline hs-Tnl, median (IQR) & $5(3,128)$ & $3(3,7)$ & 0.116 \\
\hline hs-Tnl >37 ng/L, n\% & $2 / 9$ & $4 / 61$ & Fisher's exact test 0.168 \\
\hline NT-proBNP, median (IQR) & $134(88,298)$ & $83(48,124)$ & 0.114 \\
\hline NT-proBNP >125 pg/mL, n\% & $6 / 10$ & $15 / 64$ & Fisher's exact test $0.026^{\star}$ \\
\hline
\end{tabular}

Mean (SD) unless stated otherwise.

${ }^{*} \mathrm{p}<0.05$.

BSA, body surface area; CMR, cardiovascular magnetic resonance; CV, cardiovascular; ECV, extracellular-volume fraction; EDV, enddiastolic volume; EF, ejection fraction; ESV, end-systolic volume; hs-Tnl, high-sensitivity troponin I; LGE, late gadolinium enhancement; LV, left ventricular; MBF, myocardial blood flow; MPR, myocardial perfusion reserve; NT-proBNP, N-terminal pro-brain natriuretic peptide; SSc, systemic sclerosis; SV, stroke volume.

LGE or LGE $\%$ and CV outcomes $(\mathrm{OR}=0.481, \mathrm{p}=0.508$; $\mathrm{OR}=1.003, \mathrm{p}=0.990$, respectively) at univariate analysis. A predictive model was built using penalised likelihood estimation that included CMR variables and cardiac biomarkers. NT-proBNP above the normal threshold was the only variable associated with the development of $\mathrm{CV}$ outcomes $(\mathrm{OR}=5.335, \mathrm{p}=0.040)$ although an $\mathrm{ECV}$ above $29 \%$ almost reached similar strength $(\mathrm{OR}=4.347$, $\mathrm{p}=0.073)($ table 4$)$.

\section{DISCUSSION}

This study evaluates the association of subclinical (CMR and blood) indicators of SSc-pHI (not wider CVD) for future development of CV events. Just under 15\% (10/74) of this cohort developed $\mathrm{CV}$ outcomes including myocarditis, systolic dysfunction and arrhythmias. NT-proBNP indicating subclinical contractile impairment associated with future $\mathrm{CV}$ outcomes, with a similar trend of an imaging marker indicative of diffuse fibrosis (measured as higher ECV on CMR).

A significant proportion of unselected patients with SSc have subclinical cardiac involvement when sensitive methods are used. ${ }^{27-30}$ We and others have demonstrated the presence of focal and diffuse fibrosis as well as microvascular impairment on CMR. ${ }^{14} 1831$ The prognostic implications of such subclinical CMR abnormalities are however not established. Several cross-sectional studies demonstrate an association between LGE burden and ventricular arrhythmia as assessed by 24 hour Holter ECG, ${ }^{32-34}$ while other studies do not confirm these findings. ${ }^{75}$ Preliminary results from our group demonstrated association of CMRECV with arrhythmias on implantable loop recording as well as higher hs-TnI and NT-proBNP levels in patients with SSc with confirmed electrophysiological abnormalities. ${ }^{15}$ 

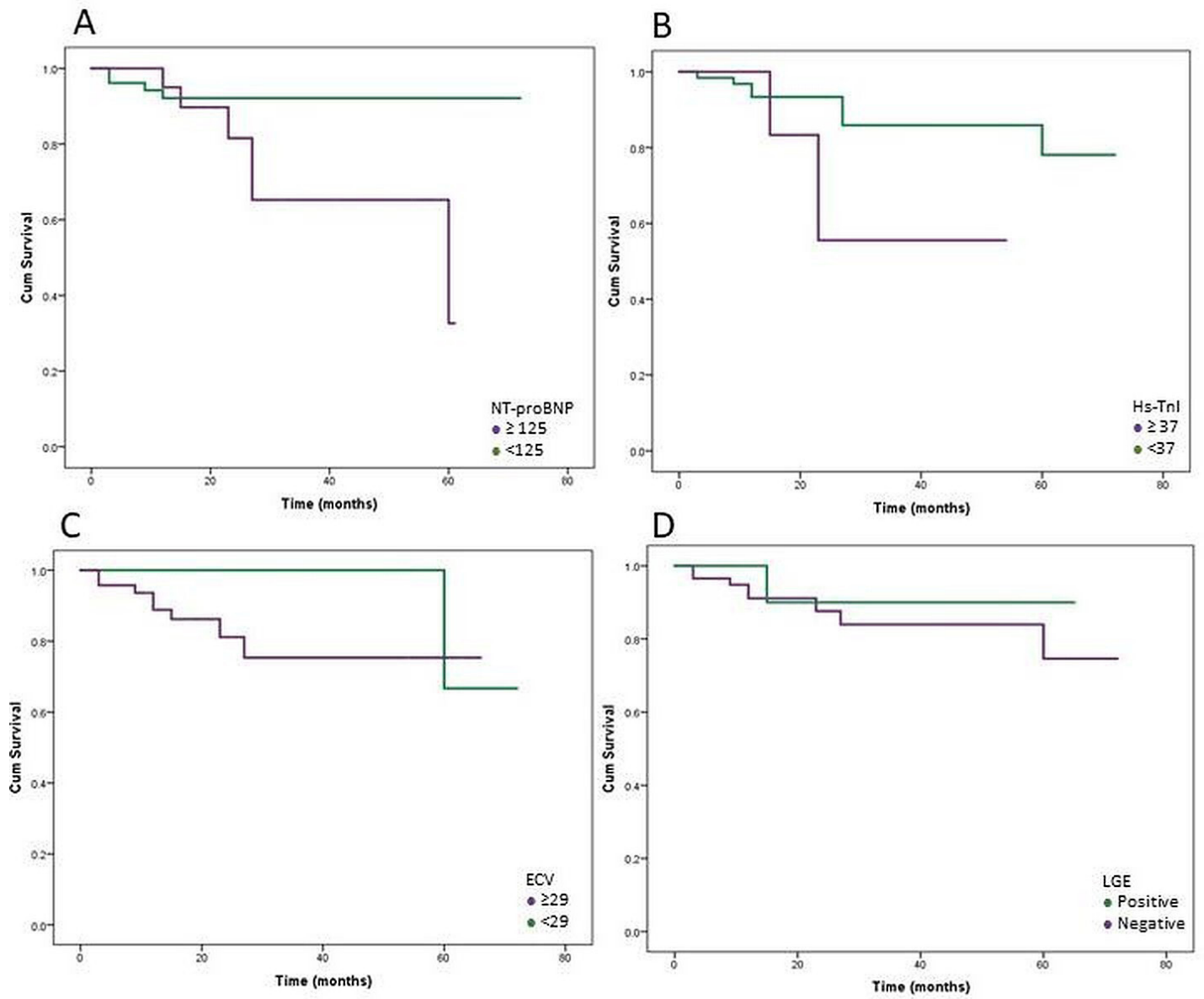

Figure 1 Time-to-event plots for cardiovascular outcomes. (A) Time-to-event plots in patients with normal and abnormal NTproBNP. (B) Time-to-event plots in patients with normal and abnormal hs-Tnl. (C) Time-to-event plots in patients with normal and abnormal ECV. (D) Time-to-event plots in patients with and without LGE. ECV, extracellular volume; hs-Tnl, high-sensitivity troponin I; LGE, late gadolinium enhancement; NT-proBNP, N-terminal pro-brain natriuretic peptide.

The current study sought to build on this further to explore the value of CMR and cardiac biomarkers in the development of a future prognostic model for predicting SSc-pHI CV outcomes. Several studies have investigated the prognostic value of CMR for predicting CV outcomes in a longitudinal cohort. ${ }^{34} 36$ The Scleroderma Arrhythmia Clinical Utility Study included 150 patients with SSc and reported predictive value of increased T2 ratio, a marker of myocardial oedema and $\mathrm{LGE} \%$ for the development of ventricular rhythm disturbances and sudden cardiac death in SSc. Another study in 40 consecutive patients with SSc that had T1 mapping and diffusion-weighted imaging CMR showed association of $\mathrm{T} 1$ native with the occurrence of $\mathrm{CV}$ events ${ }^{37}$ while Bordonaro et al, looking retrospectively at a symptomatic SSc cohort reported LGE, T1 native and ECV as independent predictors of CV events. ${ }^{38}$ These studies included patients with both primary and secondary CVD, capturing real-life cohorts. Our study is focusing on primary SSc cardiac pathology; in order to understand pathophysiology of SSc-pHI and to inform on risk stratification. We thus excluded patients with any prior diagnosis of heart disease, that comprised cardiomyopathies, heart failure, valve disease, PAH,
IHD, diabetes and more than two CV risk factors at baseline and defined CV outcomes that were attributable to SSc-pHI. Fourteen per cent of the patients developed CV outcomes consistent with SSc-pHI including myocarditis, supraventricular and ventricular arrhythmias. NT-proBNP associated with the development of $\mathrm{CV}$ outcomes, indicating that measures of subclinical myocardial contractile impairment in a cohort with otherwise no clinical suggestion of cardiac involvement, precedes the development of $\mathrm{CV}$ outcomes in SSc. Given the ease and low cost of blood biomarkers, this finding will provide an accessible means of stratifying for SSc-pHI. Hs-TnI did not clearly associate with CV outcomes. This is perhaps expected as hs-TnI is an indicator of myocardial injury, associated with acute CV events. The time of serum hs-TnI sampling in this asymptomatic cohort is thus particularly relevant as may be the relatively small sample size, limiting ability of prediction. A longitudinal study with more frequent sampling for hs-TnI (and NT-proBNP) would clarify this further. Several other studies have also suggested an association of higher NT-proBNP and hs-Tn levels with the development of $\mathrm{CV}$ events in patients with SSc. ${ }^{15} 39$ No studies however explored the value of 
Table 4 Penalised logistic regression analysis for predicting cardiovascular outcomes including cardiac biomarkers and CMR parameters

\begin{tabular}{lccl}
\hline Variable & Estimate & P value & OR \\
\hline Cardiac biomarkers & & & \\
\hline NT-proBNP $>125 \mathrm{pg} / \mathrm{mL}$ & 1.674 & $0.040^{*}$ & 5.335 \\
\hline hs-Tnl $>37 \mathrm{ng} / \mathrm{L}$ & 0.587 & 0.625 & 1.798 \\
\hline CMR parameters & & & \\
\hline LGE & -1.234 & 0.273 & 0.290 \\
\hline T1 native & -0.007 & 0.171 & 0.993 \\
\hline ECV \% (>29) & 1.469 & 0.073 & 4.347 \\
\hline MPR \% & 0.029 & 0.947 & 1.029 \\
\hline LVEDV/BSA & -0.041 & 0.475 & 0.959 \\
\hline LVESV/BSA & 0.155 & 0.230 & 1.167 \\
\hline LVEF & 0.185 & 0.222 & 1.203 \\
\hline LV mass/BSA & 0.049 & 0.298 & 1.050 \\
\hline
\end{tabular}

${ }^{*} \mathrm{p}<0.05$.

BSA, body surface area; CMR, cardiovascular magnetic resonance; ECV, extracellular-volume fraction; EDV, enddiastolic volume; EF, ejection fraction; ESV, end-systolic volume; hs-Tnl, high-sensitivity troponin I; LGE, late gadolinium enhancement; LV, left ventricular; MPR, myocardial perfusion reserve; NT-proBNP, N-terminal pro-brain natriuretic peptide.;

cardiac biomarkers in association with CMR indices for the development of SSc-pHI in a longitudinal cohort.

ECV, suggestive of diffuse myocardial fibrosis showed a trend for association with $\mathrm{CV}$ outcomes. Eight of nine subjects with a CV outcome had an ECV above the normal threshold (>29\%) -although raised ECV was common also in patients without CV events. Timeto-event plots showed that patients with higher ECV had more $\mathrm{CV}$ outcomes. Increasing data from ischaemic and non-ischaemic cardiomyopathies demonstrate the prognostic relevance of $\mathrm{T} 1$ mapping indices (native $\mathrm{T} 1$ and $\mathrm{ECV}$ ) in predicting $\mathrm{CV}$ events and we have recently documented the presence of higher ECV in patients with SSc and significant arrhythmias. ${ }^{15} 4041$ Fibrosis, in particular diffuse fibrosis might thus represent the substrate for the development of CV outcomes and a sensitive marker in the early detection of future SSc-pHI.

Only 1 of the 14 patients with focal fibrosis on LGE had a CV outcome (ie, of 10 subjects with a CV outcome, only 1 subject showed focal fibrosis LGE). The relatively small sample size and event ratio as well as the relatively low extent of fibrosis in our study likely accounts for the lack of an association between CV outcome and LGE, which is inconsistent with other studies. However, the extent and location of LGE need to be taken into consideration as these are important determinants for the development of arrhythmias and CV events in both ischaemic and non-ischaemic cardiomyopathies. ${ }^{42} 43$ These data imply scar mass may be more modest in SScpHI and thus of limited utility.
Both lower and higher LV mass have been reported in different IMID ${ }^{44-46}$; in our study, patients with confirmed SSc-pHI CV outcomes had greater LV mass, although this did not reach significance. Increased LV mass and LV hypertrophy are recognised as predictors of sudden cardiac death and arrhythmia in different cardiac pathologies. ${ }^{47}{ }^{48}$ Further research is needed to clarify the significance of increased LV mass in SSc.

This study also investigated the association between myocardial perfusion and the development of $\mathrm{CV}$ outcomes, with no association demonstrated. The significance of myocardial microvascular impairment in SSc needs to be further addressed in longer follow-up studies.

This study has some limitations. While this is a goodsized longitudinal follow-up study in the context of the existing literature, larger cohorts and longer follow-up with more events will be needed to conclusively determine the prognostic value of abnormal CMR indices. An inception cohort and/or more patients with early disease would be more representative to avoid selection bias and potentially miss CV outcomes occurring early in the disease. T2-weighted imaging, a common sequence used for the detection of myocardial inflammation was not included in the CMR protocol, however, T1 mapping, proven to have superior diagnostic accuracy in detecting myocardial oedema and fibrosis was instead used. ${ }^{49} 50$ We combined two cohorts that had different monitoring methods. The majority of patients with arrhythmia were from the smaller cohort with ILR monitoring and were asymptomatic. CV outcomes may have been missed in the non-ILR group, potentially underestimating the event rates of the study. Most of the observed endpoints were arrhythmias occurring in the atria. Despite the known link between myocardial disease and atrial arrhythmia, this observation may partly explain the lack of correlation between focal fibrosis as measured with LGE and the clinical endpoints in this study. Blood biomarkers and ECV appear more predictive of atrial arrhythmias as they are more general markers of cardiac disease.

In conclusion, this study provides initial evidence for a prognostic risk model of clinical SSc-pHI and identifies in particular NT-proBNP and potentially diffuse myocardial fibrosis by CMR as associating with future CV events. Larger, longitudinal studies are required to confirm the findings and inform risk stratification for future development of CV outcomes of SSc-pHI.

\section{Author affiliations}

${ }^{1}$ Leeds Institute of Rheumatic and Musculoskeletal Medicine, University of Leeds Faculty of Medicine and Health, Leeds, UK

${ }^{2}$ National Institute for Health Research, Leeds Biomedical Research Centre, Leeds, UK

${ }^{3}$ Department of Biomedical Imaging Science, Leeds Institute of Cardiovascular and Metabolic Medicine, University of Leeds, Leeds, UK

${ }^{4}$ Leeds Teaching Hospitals NHS Trust Department of Cardiology, Leeds, UK

${ }^{5}$ Faculty of Business Economics and Law, University of Surrey, Guildford, UK 
${ }^{6}$ Department of Biochemical Diagnostics, Medical University of Lublin, Lublin, Lubelskie, Poland

${ }^{7}$ Centre for Musculoskeletal Research, School of Biological Sciences, Faculty of Biology, Medicine \& Health, University of Manchester, Manchester, UK ${ }^{8}$ NIHR Manchester Biomedical Research Centre, Manchester University NHS Foundation Trust, Manchester Academic Health Science Centre, University of Manchester, Manchester, UK

Contributors Study concept and design: MHB, SP, FdG and RBD. Drafting the manuscript: RBD. Revising the article and intellectual content: L-AB, MHB, SP, BE, AK, A-MHD, GF, GA, HD, AB, JPG, JB, DS and FdG. Data collection: RBD, L-AB, BE and GF. Analysis and interpretation of the data: RBD, A-MHD and MHB. RBD and MHB have full access to all data and take responsibility for the integrity of the data. MHB is responsible for the overall content and acts as guarantor for this manuscript. All authors revised the paper and approved the final version.

Funding This work was partly supported by Scleroderma and Raynaud's UK. RBD and $\mathrm{L}-\mathrm{AB}$ have been funded by the ACORN charity. RBD has been also funded by the Charitable Foundation Fellowship, Leeds Teaching Hospital. JB is funded by a National Institute of Health Research (NIHR) Clinical Lecturship (ICA-CL-201602-017). GF was funded by a NIHR grant (number: $11 / 117 / 27$ ). SP is funded by a British Heart Foundation Personal Chair (CH/16/2/32089).

Competing interests None declared.

Patient consent for publication Not applicable.

Ethics approval The Yorkshire and The Humber—Leeds East Research Ethics Committee: REC 12/YH/0298, RR10/9608.

Provenance and peer review Not commissioned; externally peer reviewed.

Data availability statement The datasets generated during and/or analysed during the current study are available from the corresponding author on reasonable request.

Open access This is an open access article distributed in accordance with the Creative Commons Attribution Non Commercial (CC BY-NC 4.0) license, which permits others to distribute, remix, adapt, build upon this work non-commercially, and license their derivative works on different terms, provided the original work is properly cited, appropriate credit is given, any changes made indicated, and the use is non-commercial. See: http://creativecommons.org/licenses/by-nc/4.0/.

ORCID iD

Maya H Buch http://orcid.org/0000-0002-8962-5642

\section{REFERENCES}

1 Tyndall AJ, Bannert B, Vonk M, et al. Causes and risk factors for death in systemic sclerosis: a study from the EULAR scleroderma trials and research (EUSTAR) database. Ann Rheum Dis 2010;69:1809-15

2 Steen VD, Medsger TA. Changes in causes of death in systemic sclerosis, 1972-2002. Ann Rheum Dis 2007;66:940-4.

3 de Groote P, Gressin V, Hachulla E, et al. Evaluation of cardiac abnormalities by Doppler echocardiography in a large nationwide multicentric cohort of patients with systemic sclerosis. Ann Rheum Dis 2008;67:31-6.

4 Steen VD, Medsger TA. Severe organ involvement in systemic sclerosis with diffuse scleroderma. Arthritis Rheum 2000;43:2437-44.

5 Medsger TA, Masi AT. Survival with scleroderma. II. A life-table analysis of clinical and demographic factors in 358 male U.S. veteran patients. J Chronic Dis 1973;26:647-60.

6 Kostis JB, Seibold JR, Turkevich D, et al. Prognostic importance of cardiac arrhythmias in systemic sclerosis. Am J Med 1988;84:1007-15.

7 Rodríguez-Reyna TS, Morelos-Guzman M, Hernández-Reyes P, et al. Assessment of myocardial fibrosis and microvascular damage in systemic sclerosis by magnetic resonance imaging and coronary angiotomography. Rheumatology 2015;54:647-54.

8 Mueller KAL, Mueller II, Eppler D, et al. Clinical and histopathological features of patients with systemic sclerosis undergoing endomyocardial biopsy. PLoS One 2015;10:e0126707.

9 Mavrogeni SI, Bratis K, Karabela G, et al. Cardiovascular magnetic resonance imaging clarifies cardiac pathophysiology in early, asymptomatic diffuse systemic sclerosis. Inflamm Allergy Drug Targets 2015;14:29-36.

10 Follansbee WP, Miller TR, Curtiss El, et al. A controlled clinicopathologic study of myocardial fibrosis in systemic sclerosis (scleroderma). J Rheumatol 1990;17:656-62.
11 Allanore Y, Borderie D, Avouac J, et al. High N-terminal probrain natriuretic peptide levels and low diffusing capacity for carbon monoxide as independent predictors of the occurrence of precapillary pulmonary arterial hypertension in patients with systemic sclerosis. Arthritis Rheum 2008;58:284-91.

12 Ntusi NAB, Piechnik SK, Francis JM, et al. Subclinical myocardial inflammation and diffuse fibrosis are common in systemic sclerosis-a clinical study using myocardial T1-mapping and extracellular volume quantification. J Cardiovasc Magn Reson 2014;16:21.

13 Lee DC, Hinchcliff ME, Sarnari R, et al. Diffuse cardiac fibrosis quantification in early systemic sclerosis by magnetic resonance imaging and correlation with skin fibrosis. J Scleroderma Relat Disord 2018;3:159-69.

14 Gyllenhammar T, Kanski M, Engblom H, et al. Decreased global myocardial perfusion at adenosine stress as a potential new biomarker for microvascular disease in systemic sclerosis: a magnetic resonance study. BMC Cardiovasc Disord 2018;18:16.

15 Bissell L-A, Dumitru RB, Erhayiem B, et al. Incidental significant arrhythmia in scleroderma associates with cardiac magnetic resonance measure of fibrosis and hs-Tnl and NT-proBNP. Rheumatology 2019;58:1221-6.

16 Avouac J, Meune C, Chenevier-Gobeaux C, et al. Cardiac biomarkers in systemic sclerosis: contribution of high-sensitivity cardiac troponin in addition to $\mathrm{N}$-terminal pro-brain natriuretic peptide. Arthritis Care Res 2015;67:1022-30.

17 Nordin A, Svenungsson E, Björnådal L, et al. Troponin I and echocardiography in patients with systemic sclerosis and matched population controls. Scand J Rheumatol 2017;46:226-35.

18 Dumitru RB, Bissell L-A, Erhayiem B, et al. Predictors of subclinical systemic sclerosis primary heart involvement characterised by microvasculopathy and myocardial fibrosis. Rheumatology 2021;60:2934-45.

19 van den Hoogen F, Khanna D, Fransen J, et al. 2013 classification criteria for systemic sclerosis: an American College of rheumatology/ European League against rheumatism collaborative initiative. Ann Rheum Dis 2013:72:1747-55.

20 LeRoy EC, Black C, Fleischmajer R, et al. Scleroderma (systemic sclerosis): classification, subsets and pathogenesis. J Rheumatol 1988:15:202-5.

21 Erhayiem B, Pavitt S, Baxter P, et al. Coronary artery disease evaluation in rheumatoid arthritis (CADERA): study protocol for a randomized controlled trial. Trials 2014;15:436.

22 Cerqueira MD, Weissman NJ, Dilsizian V, et al. Standardized myocardial segmentation and nomenclature for tomographic imaging of the heart. A statement for healthcare professionals from the cardiac imaging Committee of the Council on clinical cardiology of the American heart association. Circulation 2002;105:539-42.

23 McAlindon E, Pufulete M, Lawton C, et al. Quantification of infarct size and myocardium at risk: evaluation of different techniques and its implications. Eur Heart J Cardiovasc Imaging 2015;16:738-46.

24 Miller CA, Naish JH, Bishop P, et al. Comprehensive validation of cardiovascular magnetic resonance techniques for the assessment of myocardial extracellular volume. Circ Cardiovasc Imaging 2013;6:373-83.

25 Biglands JD, Magee DR, Sourbron SP, et al. Comparison of the diagnostic performance of four quantitative myocardial perfusion estimation methods used in cardiac MR imaging: CE-MARC substudy. Radiology 2015;275:393-402.

26 Kawel-Boehm N, Maceira A, Valsangiacomo-Buechel ER, et al. Normal values for cardiovascular magnetic resonance in adults and children. J Cardiovasc Magn Reson 2015;17:29.

27 Yiu KH, Schouffoer AA, Marsan NA, et al. Left ventricular dysfunction assessed by speckle-tracking strain analysis in patients with systemic sclerosis: relationship to functional capacity and ventricular arrhythmias. Arthritis Rheum 2011;63:3969-78.

28 Di Cesare E, Battisti S, Di Sibio A, et al. Early assessment of sub-clinical cardiac involvement in systemic sclerosis (SSC) using delayed enhancement cardiac magnetic resonance (CE-MRI). Eur Radiol 2013;82:e268-73.

29 Thuny F, Lovric D, Schnell F, et al. Quantification of myocardial extracellular volume fraction with cardiac MR imaging for early detection of left ventricle involvement in systemic sclerosis. Radiology 2014;271:373-80.

30 Poindron V, Chatelus E, Canuet M, et al. T1 mapping cardiac magnetic resonance imaging frequently detects subclinical diffuse myocardial fibrosis in systemic sclerosis patients. Semin Arthritis Rheum 2020;50:128-34.

31 Hachulla A-L, Launay D, Gaxotte V, et al. Cardiac magnetic resonance imaging in systemic sclerosis: a cross-sectional observational study of 52 patients. Ann Rheum Dis 2009:68:1878-84. 
32 Gargani L, Todiere G, Guiducci S, et al. Early Detection of Cardiac Involvement in Systemic Sclerosis: The Added Value of Magnetic Resonance Imaging. JACC Cardiovasc Imaging 2019;12:927-8.

33 Tzelepis GE, Kelekis NL, Plastiras SC, et al. Pattern and distribution of myocardial fibrosis in systemic sclerosis: a delayed enhanced magnetic resonance imaging study. Arthritis Rheum 2007;56:3827-36.

34 Mavrogeni S, Gargani L, Pepe A, et al. Cardiac magnetic resonance predicts ventricular arrhythmias in scleroderma: the scleroderma arrhythmia clinical utility study (SAnCtUS). Rheumatology 2020;59:1938-48

35 Muresan L, Oancea I, Mada RO, et al. Relationship between ventricular arrhythmias, conduction disorders, and myocardial fibrosis in patients with systemic sclerosis. J Clin Rheumatol 2018;24:25-33.

36 Rodríguez-Reyna TS, Rosales-Uvera SG, Kimura-Hayama E, et al. Myocardial fibrosis detected by magnetic resonance imaging, elevated U-CRP and higher mRSS are predictors of cardiovascular complications in systemic sclerosis (SSC) patients. Semin Arthritis Rheum 2019;49:273-8.

37 Terrier B, Dechartres A, Gouya $\mathrm{H}$, et al. Cardiac Intravoxel incoherent motion diffusion-weighted magnetic resonance imaging with T1 mapping to assess myocardial perfusion and fibrosis in systemic sclerosis: association with cardiac events from a prospective cohort study. Arthritis Rheumatol 2020;72:1571-80.

38 Bordonaro V, Bivort D, Dresselaers T, et al. Myocardial T1 mapping and extracellular volume quantification as novel biomarkers in risk stratification of patients with systemic sclerosis. Clin Radiol 2021;76:162.e1-162.e8.

39 Bosello S, De Luca G, Berardi G, et al. Cardiac troponin T and NT-proBNP as diagnostic and prognostic biomarkers of primary cardiac involvement and disease severity in systemic sclerosis: a prospective study. Eur J Intern Med 2019;60:46-53.

40 Puntmann VO, Carr-White G, Jabbour A, et al. Native T1 and ECV of Noninfarcted Myocardium and Outcome in Patients With Coronary Artery Disease. J Am Coll Cardiol 2018;71:766-78.
41 Wong TC, Piehler K, Meier CG, et al. Association between extracellular matrix expansion quantified by cardiovascular magnetic resonance and short-term mortality. Circulation 2012;126:1206-16.

42 Leyva F, Taylor RJ, Foley PWX, et al. Left ventricular midwall fibrosis as a predictor of mortality and morbidity after cardiac resynchronization therapy in patients with nonischemic cardiomyopathy. J Am Coll Cardiol 2012;60:1659-67.

43 Alexandre J, Saloux E, Dugué AE, et al. Scar extent evaluated by late gadolinium enhancement CMR: a powerful predictor of long term appropriate ICD therapy in patients with coronary artery disease. $J$ Cardiovasc Magn Reson 2013;15:12.

44 Giles JT, Malayeri AA, Fernandes V, et al. Left ventricular structure and function in patients with rheumatoid arthritis, as assessed by cardiac magnetic resonance imaging. Arthritis Rheum 2010;62:940-51

45 Greulich S, Mayr A, Kitterer D, et al. T1 and T2 mapping for evaluation of myocardial involvement in patients with ANCAassociated vasculitides. J Cardiovasc Magn Reson 2017;19:6.

46 Aslam F, Bandeali SJ, Khan NA, et al. Diastolic dysfunction in rheumatoid arthritis: a meta-analysis and systematic review. Arthritis Care Res 2013:65:534-43.

47 Stevens SM, Reinier K, Chugh SS. Increased left ventricular mass as a predictor of sudden cardiac death: is it time to put it to the test? Circ Arrhythm Electrophysiol 2013;6:212-7.

48 Turakhia MP, Schiller NB, Whooley MA. Prognostic significance of increased left ventricular mass index to mortality and sudden death in patients with stable coronary heart disease (from the heart and soul study). Am J Cardiol 2008;102:1131-5.

49 Nazir SA, Shetye A, Khan JN, et al. Comparison of T1-mapping and T2-weighted imaging for diagnostic oedema assessment in STsegment elevation myocardial infarction. Journal of Cardiovascular Magnetic Resonance 2016;18:Q9.

50 Ferreira VM, Piechnik SK, Dall'Armellina E, et al. T(1) mapping for the diagnosis of acute myocarditis using CMR: comparison to T2weighted and late gadolinium enhanced imaging. JACC CardiovasC Imaging 2013;6:1048-58. 